\title{
Induction of Antibodies and T Cell Responses by a Recombinant Influenza Virus Carrying an HIV-1 Tat $\Delta_{51-59}$ Protein in Mice
}

\author{
B. Garulli, ${ }^{1,2}$ G. Di Mario, ${ }^{1}$ M. G. Stillitano, ${ }^{1}$ D. Compagnoni, ${ }^{3}$ F. Titti, ${ }^{3}$ A. Cafaro, ${ }^{3}$ \\ B. Ensoli, ${ }^{3}$ Y. Kawaoka, ${ }^{4,5,6}$ and M. R. Castrucci ${ }^{1}$ \\ ${ }^{1}$ Department of Infectious, Parasitic and Immune-Mediated Diseases, Istituto Superiore di Sanità, 00161 Rome, Italy \\ ${ }^{2}$ Department of Biology and Biotechnology "Charles Darwin", Sapienza University of Rome, 00185 Rome, Italy \\ ${ }^{3}$ National AIDS Center, Istituto Superiore di Sanità, 00161 Rome, Italy \\ ${ }^{4}$ Department of Pathobiological Sciences, School of Veterinary Medicine, University of Wisconsin-Madison, Madison, WI 53711, USA \\ ${ }^{5}$ Division of Virology, Department of Microbiology and Immunology and International Research Center for Infectious Diseases, \\ Institute of Medical Science, University of Tokyo, Tokyo 108-8639, Japan \\ ${ }^{6}$ Infection-Induced Host Responses Project, Exploratory Research for Advanced Technology, Saitama 332-0012, Japan \\ Correspondence should be addressed to M. R. Castrucci; mariarita.castrucci@iss.it
}

Received 26 February 2014; Revised 17 April 2014; Accepted 22 April 2014; Published 14 May 2014

Academic Editor: Wen-Quan Zou

Copyright (C) 2014 B. Garulli et al. This is an open access article distributed under the Creative Commons Attribution License, which permits unrestricted use, distribution, and reproduction in any medium, provided the original work is properly cited.

\begin{abstract}
Recombinant influenza viruses hold promise as vectors for vaccines to prevent transmission of mucosal pathogens. In this study, we generated a recombinant WSN/Tat $\Delta_{51-59}$ virus in which Tat protein lacking residues 51 to 59 of the basic domain was inserted into the N-terminus of the hemagglutinin (HA) of A/WSN/33 virus. The Tat $\Delta_{51-59}$ insertion into the viral HA caused a 2-log reduction in viral titers in cell culture, compared with the parental A/WSN/33 virus, and severely affected virus replication in vivo. Nevertheless, Tat-specific antibodies and $\mathrm{T}$ cell responses were elicited upon a single intranasal immunization of BALB/c mice with WSN/Tat $\Delta_{51-59}$ virus. Moreover, Tat-specific immune responses were also detected following vaccine administration via the vaginal route. These data provide further evidence that moderately large HIV antigens can be delivered by chimeric HA constructs and elicit specific immune responses, thus increasing the options for the potential use of recombinant influenza viruses, and their derivatives, for prophylactic and therapeutic vaccines.
\end{abstract}

\section{Introduction}

The continuous spread of HIV-1 calls for efforts to develop vaccines that are protective and capable of eliciting both cellular and humoral immunity at systemic and mucosal sites. Several studies suggest that the abatement of HIV replication at mucosal surfaces is likely to be essential for the success of a prophylactic vaccine and that the local induction of high levels of high-avidity mucosal CD8+ T cells with antigen protective specificities may be able to prevent, at early times of infection, the steady and massive spread of virus from the mucosa into the systemic circulation [1-4]. Antienvelope responses can play an important protective role, and antibodies to early HIV regulatory gene products, including Tat, Rev, and Nef, are expected to impact HIV acute infection.
In particular, the Tat protein is produced very early after HIV-1 infection and is necessary for viral gene expression and disease progression [5]. This protein is well conserved among the circulating HIV-1 clades, and cross-sectional and longitudinal studies of natural infection suggest that the presence of an anti-Tat immune response correlates with asymptomatic infections and a slower progression to disease [6-8]. It has been also shown that Tat-specific cytotoxic $\mathrm{T}$ lymphocytes (CTL) play a role in controlling the early virus infection [9]. For these reasons, a native Tat protein-based vaccine has been developed and tested in human clinical trials [10-13].

Influenza A viruses are strong inducers of humoral and cellular immune responses, and live recombinant influenza viruses have proven to be suitable vectors to present foreign 
antigens to the immune system in mice. Effective immune responses against foreign epitopes recognized in the context of class I and class II MHC molecules have been largely described upon infection by recombinant influenza viruses given via intranasal (i.n.) route [14-16]. Moreover, previous studies have shown that mucosal and systemic immune responses are also induced upon vaginal (i.vag.) immunization of progesterone-treated female mice with recombinant influenza viruses [17].

Here, we generated a recombinant influenza virus (WSN/ Tat $\Delta_{51-59}$ ) by inserting a Tat protein that lacked residues 51 to 59 of its basic domain into the N-terminus of the $\mathrm{HA}$ of $\mathrm{A} / \mathrm{WSN} / 33$ (H1N1) (WSN) virus. This coding region of tat gene was removed to prevent recombination events at the HA cleavage site and also to reduce the possibility of interactions between these basic residues and host cell factors during transit through the secretory pathway. We then characterized the humoral and cellular immune responses elicited upon a single immunization via either the i.n. or i.vag. route in mice. In this way, we could explore the strategy to accommodate moderately large HIV antigens on the viral surface as HA fusion proteins and examine their immunogenic properties, thus increasing the options for the potential use of recombinant influenza viruses as a platform for innovative vaccines. In particular, we provide evidence for a new Tat-based vaccine that may prove useful in establishing effective mucosal immunization protocols for HIV-1.

\section{Materials and Methods}

2.1. Construction of the pPolI-WSN HA/Tat ${ }_{51-59}$ Plasmid and Generation of Recombinant WSN/Tat $\Delta_{51-59}$ Virus. The nucleotide sequence encoding 86 amino acids of Tat protein (HIV-1 IIIB, BH-10 clone), flanked by ClaI and PstI restriction sites, was inserted into the cloning cassette previously generated at the $3^{\prime}$ end of the signal peptide coding sequence of the pPolI-WSN HA plasmid, yielding the pPolI-WSN HA/Tat plasmid [16]. To delete the region coding for residues 51 to 59 (KRRQRRRPP), the pPolI-WSN HA/Tat plasmid was amplified by inverse PCR and back-to-back Tat-specific primers, yielding the pPolI-WSN HA/Tat $\Delta_{51-59}$ plasmid [18]. RTPCR conditions, including primer sequences, are available on request. Plasmids constructs were sequenced to ensure that unwanted mutations were not introduced by PCR and the cloning procedures.

The recombinant influenza virus WSN/Tat $\Delta_{51-59}$ bearing the Tat $\Delta_{51-59}$ insertion in its HA was generated by use of plasmid-driven reverse genetics, as described by Neumann et al. [19]. At $48 \mathrm{~h}$ after transfection, the virus was harvested, plaque-purified in MDCK cells, and then inoculated into MDCK cells to make virus stocks. To determine the stability of the chimeric HA/Tat $\Delta_{51-59}$ gene construct, WSN/Tat $\Delta_{51-59}$ virus was passaged ten times on MDCK cells, and the presence of the insert was confirmed by direct sequencing of RT-PCR products using primers for the HA gene $\left[5^{\prime}\right.$-GGCAAAACTACTGGTCCTGT-3' (forward), 5' TACTGAGCTCAATTGCTCCC-3' (reverse)].
2.2. Western Blot Analysis. Western blot analysis of proteins obtained from virus concentrated through a $20 \%$ sucrose cushion was performed by electrophoretic transfer of the proteins from the $12 \%$ polyacrylamide gel to a PVDF-membrane (Immobilon P, Millipore) at $250 \mathrm{~mA}$ for $90 \mathrm{~min}$. After being blotted, the membrane was incubated for $2 \mathrm{~h}$ with a cocktail of monoclonal antibodies to the HAl of WSN virus or with a specific rabbit anti-Tat hyperimmune serum (diluted 1:200 in TBS) (Diatheva, Fano, Italy) for $2 \mathrm{~h}$ at room temperature. The membrane was then washed three times and incubated for $1 \mathrm{~h}$ at room temperature with HRP-goat anti-mouse or goat anti-rabbit IgG diluted 1:1.000, and immunocomplexes were detected by using the ECL-substrate system (Amersham Pharmacia Biotech).

2.3. Immunofluorescence. MDCK cells were infected with WSN/Tat $\Delta_{51-59}$ or WSN virus at a multiplicity of infection of 0.1. Twenty-four hours after infection (p.i.), the cells were washed, fixed in PBS containing 4\% paraformaldehyde, and incubated for $40 \mathrm{~min}$ with mouse monoclonal antibodies specific to the HA of WSN virus or a specific rabbit antiTat hyperimmune serum (Diatheva). After being extensively washed with PBS, cells were incubated for $30 \mathrm{~min}$ with fluorescein isothiocyanate conjugated to goat anti-mouse IgG or goat anti-rabbit IgG (Sigma), respectively.

2.4. Immunization of Mice. All animal work wasperformed in compliance with institutional guidelines and approved protocols. Female BALB/c mice were lightly anesthetized with Avertin (2,2,2-tribromoethanol) before being i.n. infected with $45 \mu \mathrm{L}$ of PBS containing $10^{5}$ plaque-forming units (PFU) of WSN/Tat $\Delta_{51-59}$ virus or $10^{3} \mathrm{PFU}$ of WSN virus. For i.vag infection, groups of mice were subcutaneously injected with $3 \mathrm{mg}$ of progesterone (Depo-Provera; Pharmacia \& Upjohn) because of the influence of the estrous cycle on viral infectivity. Five days later, the mice were synchronous in the progestinic phase and could support vaginal replication upon i.vag. injection with $10^{5} \mathrm{PFU}$ of WSN/Tat $\Delta_{51-59}$ virus or $10^{3} \mathrm{PFU}$ of WSN virus in a $10 \mu \mathrm{L}$ volume [17].

\subsection{Viral Replication in Murine Respiratory and Genital} Tracts. To assess viral replication in mouse respiratory tracts, a total of three groups of BALB/c mice ( $n=4$ /group) were immunized under anesthesia and sacrificed on days 1 , 3 , and 5 after i.n. inoculation of $10^{5} \mathrm{PFU}$ of WSN/Tat $\Delta_{51-59}$ virus. Control groups of mice were i.n. infected with $10^{3} \mathrm{PFU}$ of WSN virus and sacrificed on the same days. Lungs were aseptically removed and tissue-derived extracts were prepared by grinding the tissue samples in a homogenizer to a $10 \%$ (wt/vol) suspension in PBS. The suspensions were then centrifuged at 3,000 $\times \mathrm{g}$ for $5 \mathrm{~min}$, and the supernatants were assayed for infectious virus particles by $48 \mathrm{~h}$ incubation of serial dilutions of samples with permissive MDCK cells, followed by standard hemagglutination with chicken red blood cells. The endpoint titers were estimated in triplicate measurements by interpolation and expressed as total $\mathrm{TCID}_{50}$ /organ. 
To assess viral replication in the vaginal mucosa of mice immunized via the i.vag. route, vaginal lavages were collected daily for eight days by rinsing the vaginal cavity twice with $50 \mu \mathrm{L}$ of sterile PBS and were stored at $-80^{\circ} \mathrm{C}$ until testing. Virus titers were determined with MDCK cells, as described above.

2.6. IFN- $\gamma$-Specific ELISPOT Assay. BALB/c mice were immunized via the i.vag. or i.n. route and spleens and lymph nodes draining the respiratory tracts (mediastinal lymph nodes, MLN) and the vaginal tracts (iliac lymph nodes, ILN), respectively, were collected on day 7 p.i. and used to isolate single-cell lymphocyte populations, as previously described [20]. Antigen-specific IFN- $\gamma$-producing cells were enumerated by using a commercially available murine IFN- $\gamma$ ELISPOT kit (BD, Pharmingen). Briefly, fresh lymphocytes from distinct pools of spleens and lymph nodes were added to 96 -well ELISPOT plates precoated with the IFN- $\gamma$-specific capture antibody and incubated at $37^{\circ} \mathrm{C}$ for $24 \mathrm{~h}$ untreated or in the presence of VCF (VCFITKALGISYGRK) Tat peptide (residues 36 to 50) or influenza nucleoprotein NP147 peptide (residues 147 to 155: TYQRTRALV) $\left(\mathrm{H}-2 \mathrm{~K}^{\mathrm{d}}\right)$ [21-23]. Colored spots representing IFN- $\gamma$-releasing cells are reported as the number of spot-forming cells (SFC) per $10^{6}$ cells.

2.7. Tat-Specific T Cell Proliferation. Splenocytes $\left(4 \times 10^{5} /\right.$ $200 \mu \mathrm{L}$ ) were cultured in 96-well plates in the presence or absence of affinity-purified and biologically active Tat protein $(0.1,1,5$, or $10 \mu \mathrm{g} / \mathrm{mL})$ for 5 days at $37^{\circ} \mathrm{C} .\left[{ }^{3} \mathrm{H}\right]$ thymidine (Amersham Biosciences, UK) $(0.5 \mu \mathrm{Ci} /$ well) was added $12-15 \mathrm{~h}$ before harvesting. The stimulation index (S.I.) was calculated by dividing the mean counts/min of five wells of antigen-stimulated cells by the mean counts/min of the same cells grown in the absence of antigen.

2.8. Detection of Tat-Specific Antibodies. Serum samples were examined for antigen-specific IgG antibodies by use of an enzyme-linked immunosorbent assay (ELISA). Blood samples were collected by retroorbital plexus puncture before immunization, at 21 days after single i.n. infection with WSN or WSN/Tat $\Delta_{51-59}$ virus, and at 14 days after boost. Then, 96-well microtiter plates (Nunc-Immuno Plate MaxiSorp; Nunc Life Technologies) were coated overnight at $4^{\circ} \mathrm{C}$ with $200 \mu \mathrm{L}$ of Tat protein $(0.5 \mu \mathrm{g} / \mathrm{mL}$ in $0.05 \mathrm{M}$ carbonate buffer $\mathrm{pH}$ 9.6). The plates were washed and saturated with blocking buffer (PBS containing 0.05\% Tween 20 and 1\% BSA) for $1.5 \mathrm{~h}$ at $37^{\circ} \mathrm{C}$. After five washes, $100 \mu \mathrm{L}$ of test serum from individual mice was serially diluted in blocking buffer, added to duplicate wells, and incubated for $1.5 \mathrm{~h}$ at $37^{\circ} \mathrm{C}$. The wells were then washed again and incubated for $1.5 \mathrm{~h}$ at $37^{\circ} \mathrm{C}$ with $100 \mu \mathrm{L}$ of HRP-goat anti-mouse IgG serum (Sigma). After a final round of washes, bound antibodies were detected by the addition of $100 \mu \mathrm{L}$ of TMB (Vector), and absorbance was read at a wavelength of $450 \mathrm{~nm}$.

\section{Results}

3.1. Generation and Growth Properties of a Recombinant Influenza Virus Expressing Tat $\Delta_{51-59}$ Protein. The chimeric
$\mathrm{HA} / \mathrm{Tat} \Delta_{51-59}$ construct that lacked residues 51 to 59 of the basic region of Tat was generated, and the recombinant influenza virus, designated WSN/Tat $\Delta_{51-59}$, was rescued from cDNA by using reverse genetics [19] (Figure 1(a)). The WSN/Tat $\Delta_{51-59}$ virus was plaque-purified in MDCK and then inoculated into MDCK cells to make a virus stock. The nucleotide sequence of the viral RNA confirmed the in-frame insertion of the nucleotides encoding the Tat protein lacking residues 51 to 59 , fused at the $\mathrm{N}$-terminal end of $\mathrm{HA}$.

To determine whether the Tat sequence was incorporated into the virions, WSN and WSN/Tat $\Delta_{51-59}$ viruses were analyzed by using $12 \%$ SDS-PAGE, followed by Western Blotting with a mixture of anti-HA1 monoclonal antibodies, and a polyclonal serum specific for the Tat protein. The migration patterns of the HA0 precursor and HAl of WSN/Tat $\Delta_{51-59}$ virus were consistent with the molecular size expected for the chimeric HA/Tat $\Delta_{51-59}$ protein (Figure 1(b)). Moreover, the reactivity, or lack thereof, of the WSN/Tat $\Delta_{51-59}$ and WSN viruses, respectively, with the anti-Tat-specific serum confirmed the specificity of the insertion into the chimeric HA.

The growth kinetics of the recombinant WSN/Tat $\Delta_{51-59}$ virus in MDCK cells were reduced compared with those of the wild-type WSN virus, reaching viral titers of $10^{6.5} \mathrm{PFU} / \mathrm{mL}$ and $10^{8.2} \mathrm{PFU} / \mathrm{mL}$, respectively (Figure $3(\mathrm{a})$ ). The Tat insertion into the HA gene, revealed by PCR from different plaques after ten passages of the WSN/Tat $\Delta_{51-59}$ virus in tissue culture, indicates the stable retention and expression of the foreign antigen in the recombinant influenza virus (Figure $1(\mathrm{c})$ ). The presence of the Tat antigen in infected MDCK cells was also analyzed by immunofluorescence with a Tat-specific hyperimmune serum. A distinct immunofluorescence signal was observed in cells infected with WSN/Tat $\Delta_{51-59}$ virus, whereas no signal was detected with the control WSN virus (Figure 2).

3.2. Virulence of $W S N /$ Tat $\Delta_{51-59}$ Virus. To assess the growth properties of the recombinant WSN/Tat $\Delta_{51-59}$ virus in vivo, we first examined the ability of the virus to replicate in the respiratory tracts of $\mathrm{BALB} / \mathrm{c}$ mice. Mice were infected i.n. with $10^{5} \mathrm{PFU}$ of WSN/Tat $\Delta_{51-59}$ virus or with $10^{3} \mathrm{PFU}$ of WSN virus and the viral load was determined in the lungs (Figure 3(b)). Virus was not detected in any mice inoculated with WSN/Tat $\Delta_{51-59}$ virus at the indicated time points, and the absence of clinical and pathological signs, such as body weight loss or pneumonia, showed that WSN/Tat $\Delta_{51-59}$ virus was highly attenuated in mice. We also assessed viral replication in mice infected i.vag. with WSN or WSN/Tat $\Delta_{51-59}$ virus. Viral replication in the vaginal mucosal tissues was evident with the highest titers of WSN virus present in the vaginal washes of progesterone-treated female BALB/c mice on days 3 to 5 , whereas replication of WSN/Tat $\Delta_{51-59}$ virus was not detected at the indicated time points (Figure 3(c)). Taken together, these data show that the recombinant virus was essentially unable to cause a productive infection in vivo.

3.3. Tat-Specific $T$ Cell Responses. We next determined whether the engineered Tat $\Delta_{51-59}$ protein was immunogenic 


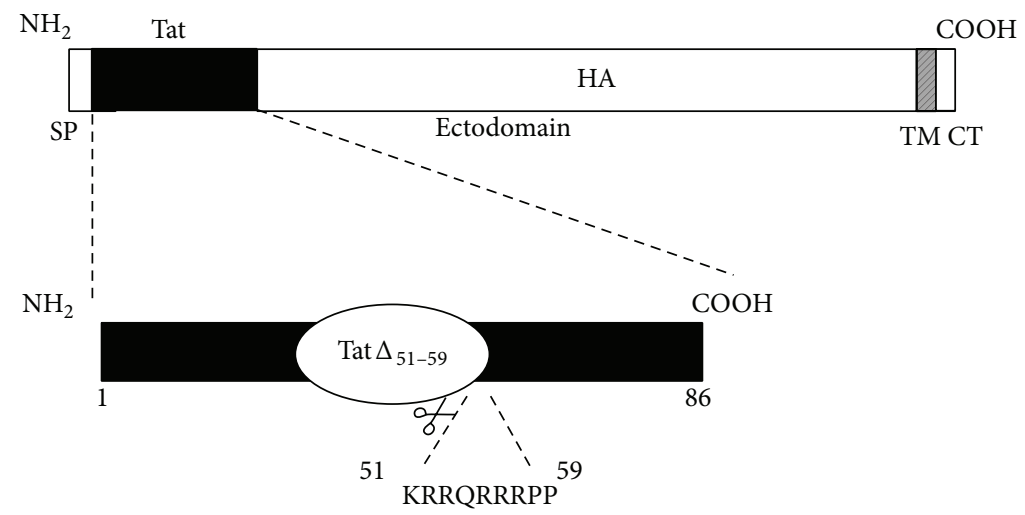

(a)

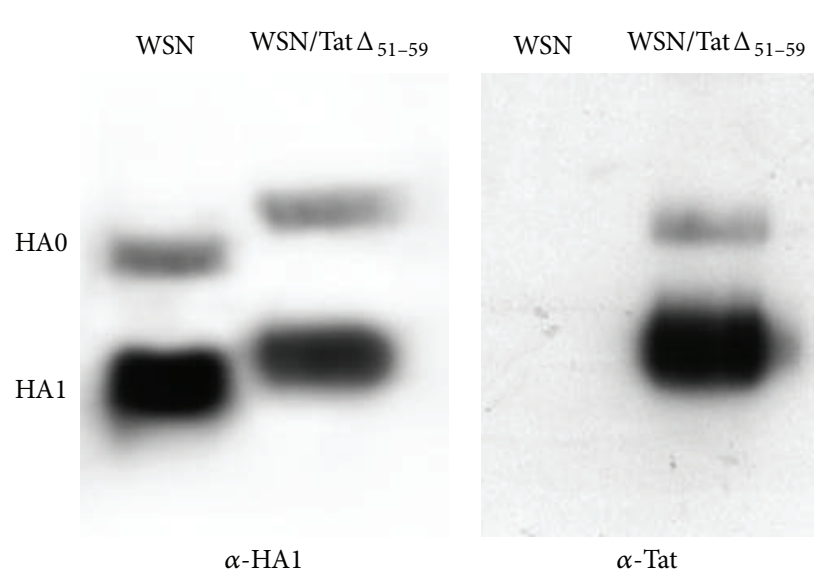

(b)

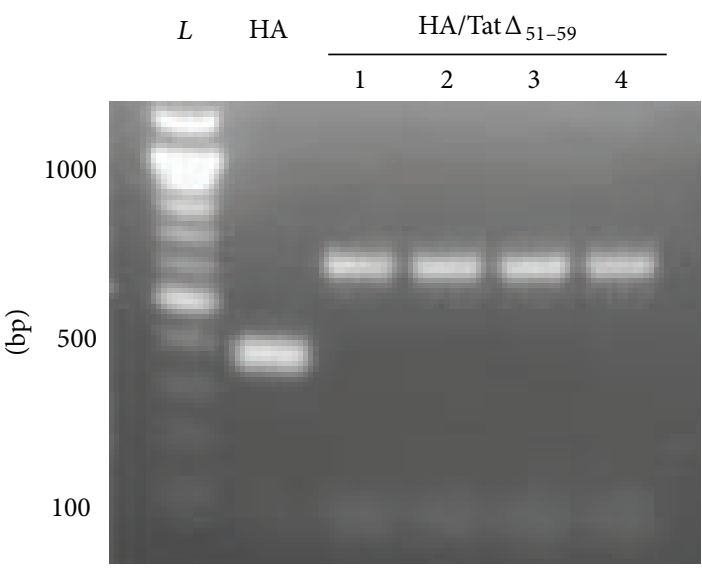

(c)

FIGURE 1: Construction and expression of WSN/Tat $\Delta_{51-59}$ virus. The coding sequence of the Tat protein of HIV IIIB (BH-10 clone), that lacked residues 51 to 59 of the basic region, was inserted into the cloning cassette of the plasmid pPolI-WSN HA, after the HA signal peptide (SP). $\mathrm{CT}, \mathrm{TM}=$ cytoplasmic, transmembrane domains of HA, respectively (a). Viral protein lysates were separated on a $12 \%$ polyacrylamide gel and analyzed in Western blots by using mouse monoclonal antibodies specific to the HA1 of WSN virus or a specific rabbit anti-Tat hyperimmune serum (b). Genetic stability of WSN/Tat $\Delta_{51-59}$ virus was determined in four different plaques by RT-PCR after six passages on MDCK cells. $L=100$ bp DNA ladder (c).

following infection with WSN/Tat $\Delta_{51-59}$ virus. Our previous studies showed that i.vag. immunization of mice with recombinant influenza viruses bearing HIV-1 antigens induces a vigorous antigen-specific CD8+ T cell immune response that is detectable in both mucosal and systemic compartments $[17,24]$. Therefore, we evaluated the $\mathrm{T}$ cell responses elicited by the WSN/Tat $\Delta_{51-59}$ virus in BALB/c mice after single i.n. or i.vag. immunization with WSN/Tat $\Delta_{51-59}$ or WSN virus, according to the immunization protocols described in the Materials and Methods. Mice were sacrificed 7 days p.i., and the frequencies of Tat-specific IFN- $\gamma$-producing T cells from regional lymph nodes and spleens were determined by use of an ex vivo ELISPOT assay with the VCF Tat peptide, which contains a $\mathrm{H}-2 \mathrm{~K}^{\mathrm{d}}$-restricted CTL epitope and a CD4+ $\mathrm{T}$ cell epitope $[22,23]$. VCF-specific IFN- $\gamma$ production was detected in cells derived from both draining lymph nodes and spleens of WSN/Tat $\Delta_{51-59}$-immunized mice (Figure 4(a)), whereas the draining lymph nodes and spleens derived from the WSN-immunized mice and naive mice did not show any HIV-specific response (data not shown). CD8+ T cell responses specific for the influenza nucleoprotein immunodominant NP147 epitope $\left(\mathrm{H}-2 \mathrm{~K}^{\mathrm{d}}\right)$ were also measured to monitor the immune response against the viral vector [21], and higher reactivity, compared with the VCF epitope, was detected. Conversely, there were 2-fold fewer NP147-specific CD8+ T cells in WSN/Tat $\Delta_{51-59}$-immunized mice, compared with those measured in WSN-immunized mice, thus confirming the low replication capacity of the recombinant virus (data not shown). Under all of the conditions tested, no reactivity was detected when we used unrelated peptides that bind to the same MHC molecules, indicating that a specific immune response was induced in the immunized mice.

The frequencies of Tat-specific IFN- $\gamma$-producing T cells were also determined after 5 days of stimulation in vitro with the VCF peptide. Specific T cell expansion and IFN- $\gamma$ production were observed only in the WSN/Tat $\Delta_{51-59^{-}}$ immunized mice (for both immunization routes), confirming the immunogenicity of the recombinant influenza virus 


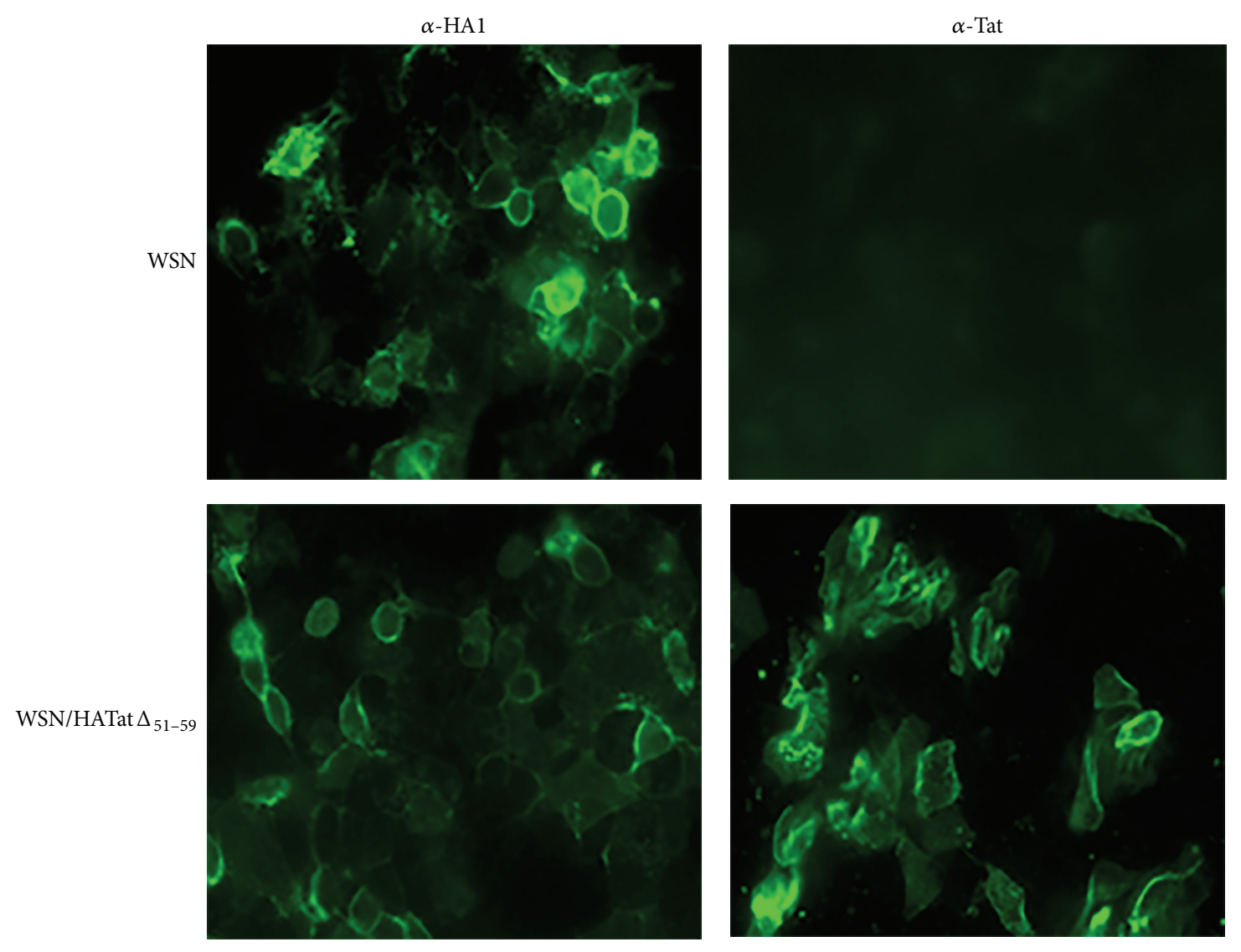

FIGURE 2: Immunofluorescence analysis. MDCK cells were infected with WSN or WSN/Tat $\Delta_{51-59}$ virus. At 24 h p.i., the cells were stained with monoclonal antibodies specific for WSN H1 HA protein or a specific rabbit anti-Tat hyperimmune serum. They were then incubated with fluorescein isothiocyanate conjugated to goat anti-mouse IgG or goat anti-rabbit IgG, respectively.

vector (Figure 4(b)). Finally, the cellular immune responses induced by a single immunization with WSN/Tat $\Delta_{51-59}$ virus were also evaluated by assessing the cell proliferation of spleen cells after in vitro stimulation with the Tat protein for 5 days. Antigen-specific and dose-dependent cell proliferation was detected in WSN/Tat $\Delta_{51-59}$-immunized mice, but not in untreated splenocytes or the lymphocytes of mice immunized with the parental WSN virus (Figure 4(c)).

3.4. Induction of Tat-Specific Antibodies by WSN/Tat $_{51-59}$ Virus Immunization. The ability to induce a humoral immune response was determined in BALB/c mice after single dose and double doses of i.n. immunization with WSN/Tat $\Delta_{51-59}$ or with WSN virus as a control. Mice were bled and sera analyzed for the presence of specific anti-Tat IgG antibodies by use of an ELISA. All mice immunized with a single dose of WSN/Tat $\Delta_{51-59}$ virus developed a detectable anti-Tat antibody response (Figure 5). A booster dose increased the antibody titers (right panel) in these mice, whereas no anti-Tat-specific response was detected in mice immunized with WSN virus. Tat delivery by the recombinant WSN/Tat $\Delta_{51-59}$ virus thus induced anti-Tat humoral responses even after a single immunization.

\section{Discussion}

Vaccines capable of inducing long-term mucosal and systemic immune responses would protect against HIV-1. Accordingly, various delivery systems and vaccination regimens have been tested in preclinical and clinical studies. Here, we successfully generated a recombinant influenza virus bearing a Tat $\Delta_{51-59}$ protein at the $\mathrm{N}$-terminus of the HA protein that was transported via the classical secretory pathway, exposed at the cell surface, and incorporated into virions. The recombinant virus was highly attenuated in mice, and a single dose was able to induce Tat-specific humoral and cellular immune responses. These findings provide evidence for the immunogenicity of the chimeric HA/Tat protein that could form the basis for a new vaccine formulation against HIV-1.

Among HIV-1 proteins, Tat is a promising antigen for the formulation of a therapeutic or prophylactic vaccine against AIDS $[25,26]$. In preclinical studies with macaques, immunization with Tat-based vaccines (both native protein and plasmid DNA) was shown to be immunogenic and effective in controlling virus replication and blocking disease onset [27-30]. Moreover, in monkeys, administration of a trimeric Env protein in the presence of a Tat subunit vaccine prevented virus spread from the portal of entry to the 


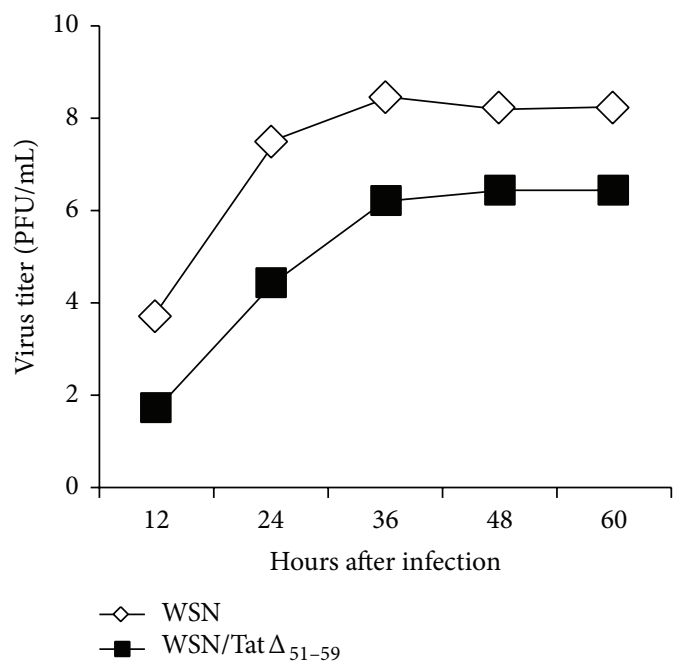

(a)



(b)

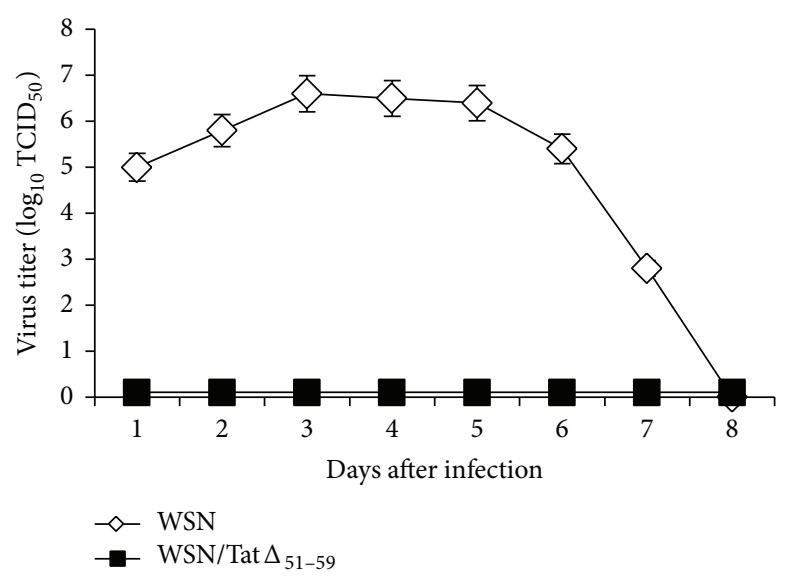

(c)

FIGURE 3: In vitro and in vivo replication of WSN/Tat $\Delta_{51-59}$ virus. Growth properties of WSN and WSN/Tat $\Delta_{51-59}$ viruses in MDCK cells. The viruses were used to inoculate MDCK cells at a multiplicity of infection of 0.01 , and at the indicated times after infection virus titers in the supernatants were determined (a). Three groups of BALB/c mice $(n=4 /$ group $)$ were i.n. infected with $10^{3}$ PFU of WSN virus and three other groups of mice $\left(n=4\right.$ /group) with $10^{5} \mathrm{PFU}$ of WSN/Tat $\Delta_{51-59}$ virus. Animals from each group were euthanized on days 1,3 , and 5 p.i., and lungs were collected from individual mice for viral titration on MDCK cells (b). Progesterone-treated mice were i.vag. infected with the above viruses, and viral titers in vaginal washes from individual mice were determined at the indicated time points by endpoint titration in MDCK cells (c). Bars represent means \pm standard deviation (SD) for four mice per group.

regional lymph nodes [31]. In humans, a Tat-based vaccine has demonstrated promising efficacy in a phase II therapeutic trial in HAART-treated subjects [13]. Thus, the development of innovative approaches of immunization designed to elicit systemic and mucosal immunity specific to Tat may improve its immunogenicity and contribute to the establishment of innovative Tat-based vaccines combined with other HIV antigens.

Recombinant influenza viruses have been used as experimental vaccine vectors to express a variety of antigens and are known to be immunogenic in mice $[14,15]$. In particular, relatively large antigens can be inserted into the HA protein and functionally expressed during infection in mice [32]. Moreover, in a recent study we showed that mucosal immunization of mice with WSN/CKG virus carrying an HIV polyepitope that contained the PCLUS3-P18 peptide in its HA induced protective immunity against challenge with recombinant vaccinia virus vPE16 expressing gp160 HIV-1 IIIB [24]. In this study, we generated WSN/Tat $\Delta_{51-59}$ virus bearing the chimeric HA/Tat protein with a 9-amino acid deletion in the basic region of Tat, which contains motifs recognized by furin-like endoproteases. This deletion prevents the rare but possible intragenic recombination events involving the multibasic residues at the cleavage site of the chimeric HA-Tat and thus the emergence of a highly pathogenic influenza virus [33]. Moreover, there is evidence that the conserved basic domain of Tat plays an important role in interactions between both viral and host cellular factors, thus mediating many multifunctional properties, such as binding to heparan sulfate proteoglycans, protein transfer across the cell membrane, 


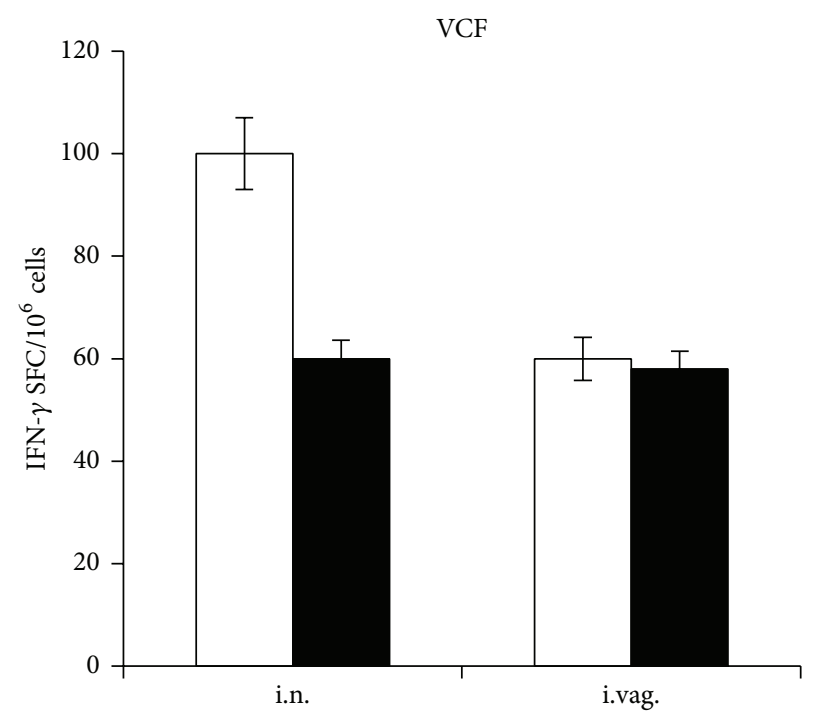

Draining LNs

Spleen

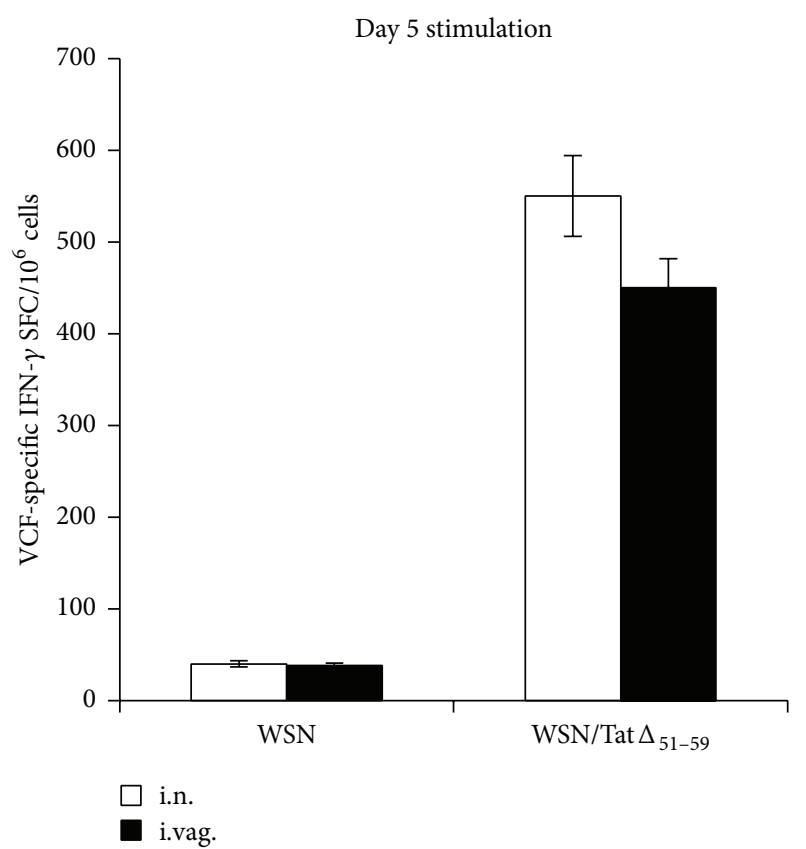

(b)

(a)
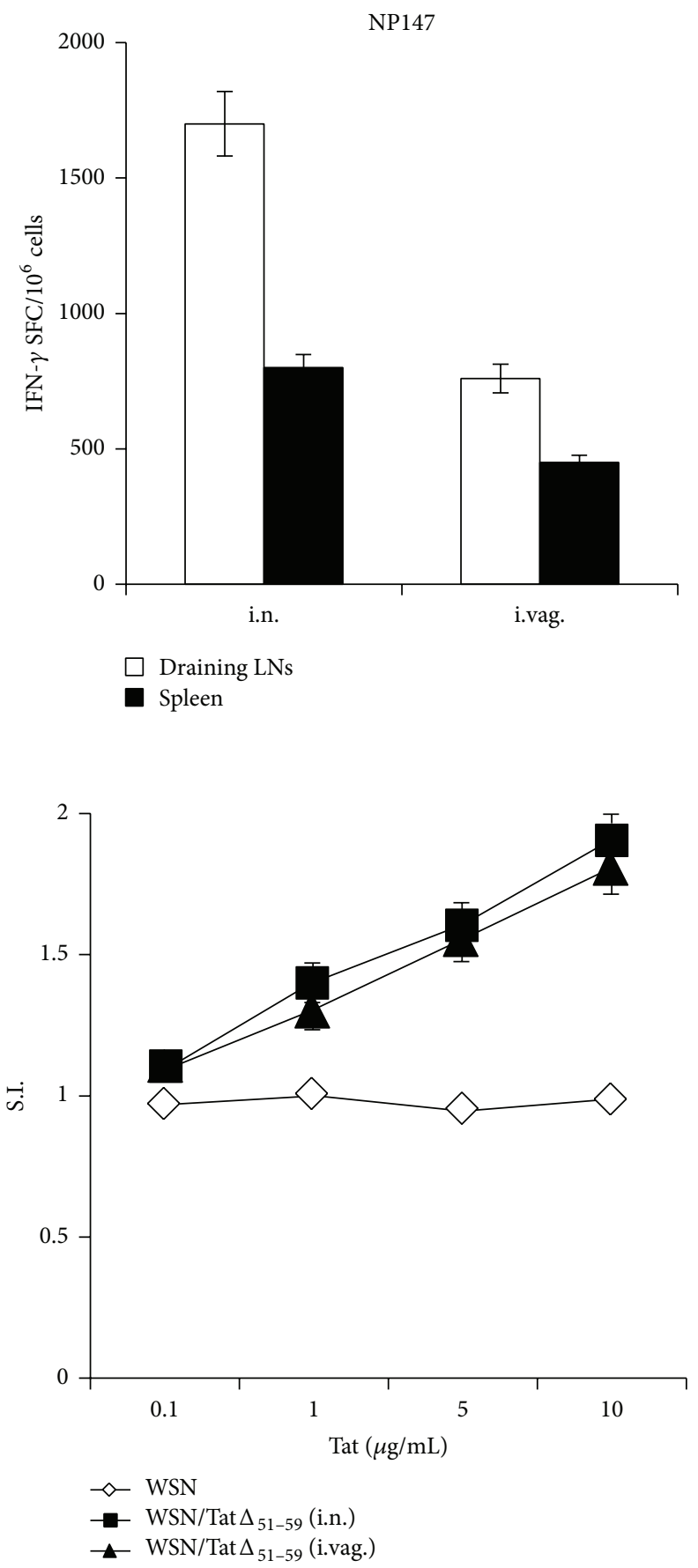

(c)

FIgURE 4: Tat-specific T cell responses in mice vaccinated with WSN/Tat $\Delta_{51-59}$ virus. BALB/c mice $(n=5 /$ group $)$ were immunized i.n. or i.vag. with WSN/Tat $\Delta_{51-59}$ virus and 7 days later Tat-specific T cell responses were measured in cells from distinct pools of draining lymph nodes, MLN and ILN, respectively, and freshly isolated splenocytes by use of an ex vivo IFN- $\gamma$ ELISPOT assay with the indicated peptides (a). Splenocytes from mice immunized with WSN/Tat $\Delta_{51-59}$ or WSN virus were cultured for 5 days in the presence of VCF and then washed for the IFN- $\gamma$ ELISPOT assay. The values reported are those obtained from stimulated cells minus the background from nonstimulated cells. Bars represent the mean values \pm SD of triplicate wells. The data are representative of three independent experiments that gave similar results (b). Splenocytes from the above mice were restimulated in the presence of different concentrations of Tat protein for 5 days and the resulting proliferation was assessed by measuring $\left[{ }^{3} \mathrm{H}\right]$ thymidine incorporation. Results are expressed as the ratio between values (averages of quintuplicates from stimulated and nonstimulated cultures). SI = stimulation index (c). 

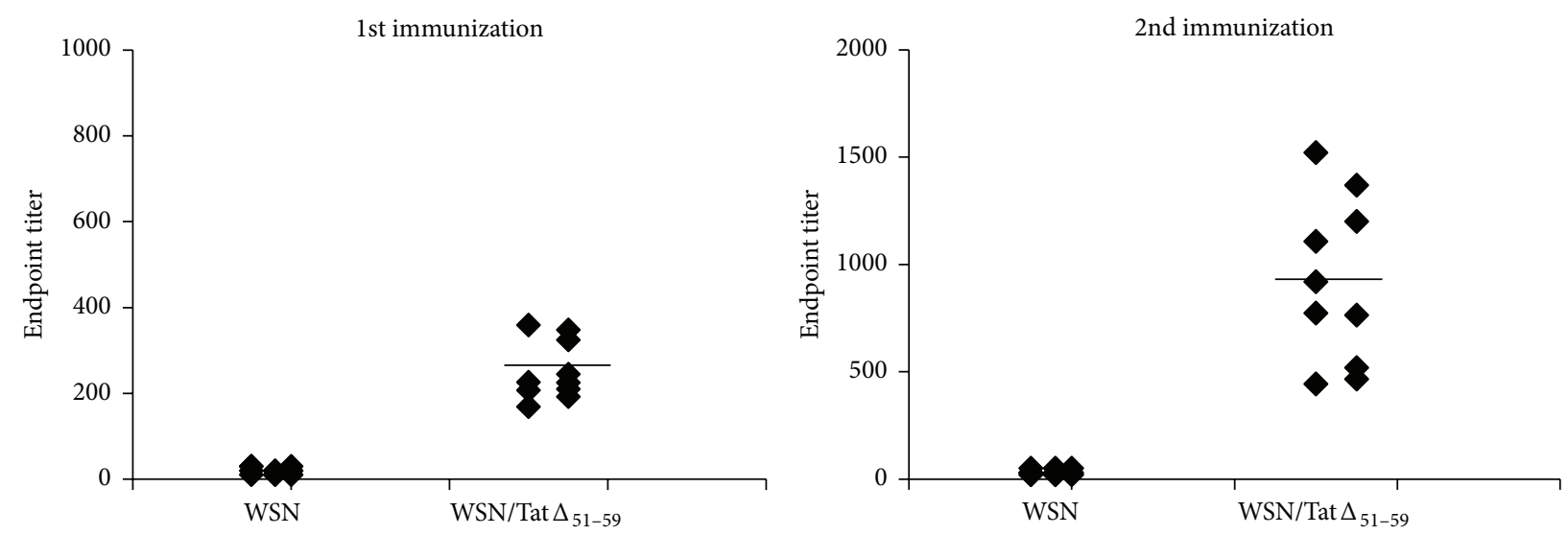

Figure 5: Humoral immune response. Mice $\left(n=10\right.$ /group) were i.n. infected with WSN or WSN/Tat $\Delta_{51-59}$ virus. Tat-specific IgG antibodies were detected at 21 days after immunization and at 14 days after boost by use of an ELISA. Results represent the endpoint values for individual mice.

nucleolar localization, and transactivating transcriptional activities [34]. By removing this basic region, we tried to prevent potential effects of the chimeric fusion protein on membrane trafficking and thus on the assembly and release of the recombinant virus.

Although we were able to generate the WSN/Tat $\Delta_{51-59}$ recombinant virus, the presence of the Tat protein affected the growth properties of the virus in tissue culture and to a greater extent in vivo. This is probably due to the Tat protein itself because the insertion of unrelated larger antigens in HA did not affect the viral replication in vitro, as described elsewhere [32]. Indeed, the relatively high numbers of CD8+ T cells specific for the influenza nucleoprotein immunodominant NP147 epitope measured in the vaccinated mice suggest that the WSN/Tat $\Delta_{51-59}$ virus was able to replicate to some extent in vivo. Although no productive virus infection was detected with the inoculum dose used, WSN/Tat virus was nevertheless able to elicit Tat-specific antibodies after a single mucosal immunization of BALB/c mice. Overall, these results support the evidence that the HA/Tat $\Delta_{51-59}$ chimeric protein was transported via the secretory pathway, exposed at the cell surface, and incorporated into virions. It is also important to point out that WSN/Tat $\Delta_{51-59}$ virus successfully induced a Tat-specific T cell response, given that Tat-specific IFN- $\gamma$ production was detectable ex vivo on fresh splenocytes of singly immunized mice. Moreover, substantial Tat-specific IFN- $\gamma$ production was also detected upon single i.vag. immunization with WSN/Tat $\Delta_{51-59}$ virus, thus confirming previous data on the efficacy of this route for recombinant influenza viruses $[17,24]$.

The ease of production of influenza virus-based immunogens and the availability of different vaccine formulations make the recombinant influenza viruses an effective vaccine platform for the delivery of foreign antigens and thus potentially suitable for heterologous prime-boost regimens. Inactivated influenza virus vaccines are excellent inducers of virus-specific serum antibodies, and the insertion of foreign antigens into the influenza HA chimeric proteins could provide potential vaccine candidates to induce HIVspecific immunity in target individuals. Additionally, one of the most interesting and feasible application would be the use of recombinant influenza virus-derived virosomes. Surface display of foreign antigens, for example, the Tat protein, in the virosomal formulations would allow the generation of Tat-specific antibodies as well as the induction of cellular immunity. In this case, even the bias of the preexisting immunity to the influenza virus in the human population would favor the Tat-specific immune responses, because memory CD4+ T cells and antibodies against influenza glycoproteins enhance the capacity of virosomes to act as carriers for the delivery of antigens for cellular immune responses [35-38]. Synthetic peptides representing $\mathrm{T}$ cell epitopes can also be packaged during the reconstitution process into recombinant influenza virus-derived virosomes engineered with chimeric HAs fused to different HIV antigens, thus providing a modular and flexible antigen delivery platform suitable for prophylactic and therapeutic vaccines. It should be noted, however, that influenza virus infection requires binding to cell surface receptors, endocytosis, and a low $\mathrm{pH}$-dependent fusion process. Thus, the design of recombinant HA proteins should always take into account the steric hindrance and conformational alterations that could interfere with the virus infection process and transport of the chimeric HA to the cell surface and consequently with the rescue of a genetically stable recombinant influenza virus.

In conclusion, our results provide a proof-of-concept for a systematic approach based on recombinant influenza viruses for promising immunogens to be further investigated as potential vaccine candidates for HIV-1.

\section{Conflict of Interests}

The authors declare that there is no conflict of interests regarding the publication of this paper. 


\section{Acknowledgments}

The authors thank Andrea Giovannelli for assistance with the animal experiments, Sabrina Tocchio for help with the paper, and Susan Watson for scientific editing. This work was funded by the AIDS project of the Ministry of Health, Italy (RF-ISS2009-1303898).

\section{References}

[1] T. Lehner, Y. Wang, M. Cranage et al., "Protective mucosal immunity elicited by targeted iliac lymph node immunization with a subunit SIV envelope and core vaccine in macaques," Nature Medicine, vol. 2, no. 7, pp. 767-775, 1996.

[2] I. M. Belyakov, Z. Hel, B. Kelsall et al., "Mucosal AIDS vaccine reduces disease and viral load in gut reservoir and blood after mucosal infection of macaques," Nature Medicine, vol. 7, no. 12, pp. 1320-1326, 2001.

[3] I. M. Belyakov, J. D. Ahlers, G. J. Nabel, B. Moss, and J. A. Berzofsky, "Generation of functionally active HIV-1 specific $\mathrm{CD}^{+} \mathrm{CTL}$ in intestinal mucosa following mucosal, systemic or mixed prime-boost immunization," Virology, vol. 381, no. 1, pp. 106-115, 2008.

[4] A. L. Ferre, P. W. Hunt, J. W. Critchfield et al., "Mucosal immune responses to HIV-1 in elite controllers: a potential correlate of immune control," Blood, vol. 113, no. 17, pp. 3978-3989, 2009.

[5] B. Romani, S. Engelbrecht, and R. H. Glashoff, "Functions of Tat: the versatile protein of human immunodeficiency virus type 1," Journal of General Virology, vol. 91, part 1, pp. 1-12, 2010.

[6] P. Reiss, J. M. A. Lange, A. de Ronde et al., "Speed of progression to AIDS and degree of antibody response to accessory gene products of HIV-1," Journal of Medical Virology, vol. 30, no. 3, pp. 163-168, 1990.

[7] M. C. Re, M. Vignoli, G. Furlini et al., "Antibodies against full-length Tat protein and some low-molecular-weight Tatpeptides correlate with low or undetectable viral load in HIV-1 seropositive patients," Journal of Clinical Virology, vol. 21, no. 1, pp. 81-89, 2001.

[8] G. Rezza, V. Fiorelli, M. Dorrucci et al., "The presence of anti-Tat antibodies is predictive of long-term nonprogression to AIDS or severe immunodeficiency: findings in a cohort of HIV-1 seroconverters," Journal of Infectious Diseases, vol. 191, no. 8, pp. 1321-1324, 2005.

[9] T. M. Allen, D. H. O'Connor, P. Jing et al., "Tat-specific cytotoxic $\mathrm{T}$ lymphocytes select for SIV escape variants during resolution of primary vimamia," Nature, vol. 407, no. 6802, pp. 386-390, 2000.

[10] B. Ensoli, V. Fiorelli, F. Ensoli et al., "The therapeutic phase I trial of the recombinant native HIV-1 Tat protein," AIDS, vol. 22, no. 16, pp. 2207-2209, 2008.

[11] O. Longo, A. Tripiciano, V. Fiorelli et al., "Phase I therapeutic trial of the HIV-1 Tat protein and long term follow-up," Vaccine, vol. 27, no. 25-26, pp. 3306-3312, 2009.

[12] B. Ensoli, V. Fiorelli, F. Ensoli et al., "The preventive phase I trial with the HIV-1 Tat-based vaccine," Vaccine, vol. 28, no. 2, pp. 371-378, 2009.

[13] B. Ensoli, S. Bellino, A. Tripiciano et al., "Therapeutic immunization with hiv-1 tat reduces immune activation and loss of regulatory t-cells and improves immune function in subjects on HAART," PLoS ONE, vol. 5, no. 11, Article ID e13540, 2010.
[14] A. García-Sastre, "Transfectant influenza viruses as antigen delivery vectors," Advances in Virus Research, vol. 55, pp. 579$597,2000$.

[15] G. Neumann and Y. Kawaoka, "Reverse genetics of influenza virus," Virology, vol. 287, no. 2, pp. 243-250, 2001.

[16] B. Garulli, G. di Mario, E. Sciaraffia, Y. Kawaoka, and M. R. Castrucci, "Immunogenicity of a recombinant influenza virus bearing both the $\mathrm{CD} 4^{+}$and $\mathrm{CD} 8^{+}$T cell epitopes of ovalbumin," Journal of Biomedicine and Biotechnology, vol. 2011, Article ID 497364, 7 pages, 2011.

[17] B. Garulli, Y. Kawaoka, and M. R. Castrucci, "Mucosal and systemic immune responses to a human immunodeficiency virus type 1 epitope induced upon vaginal infection with a recombinant influenza A virus," Journal of Virology, vol. 78, no. 2, pp. 1020-1025, 2004.

[18] H. Ochman, A. S. Gerber, and D. L. Hartl, "Genetic applications of an inverse polymerase chain reaction," Genetics, vol. 120, no. 3, pp. 621-623, 1988.

[19] G. Neumann, T. Watanabe, H. Ito et al., "Generation of influenza A viruses entirely from cloned cDNAs," Proceedings of the National Academy of Sciences of the United States of America, vol. 96, no. 16, pp. 9345-9350, 1999.

[20] B. Garulli, M. Meola, M. G. Stillitano, Y. Kawaoka, and M. R. Castrucci, "Efficient vagina-to-lower respiratory tract immune trafficking in a murine model of influenza A virus infection," Virology, vol. 361, no. 2, pp. 274-282, 2007.

[21] H. C. Bodmer, R. M. Pemberton, J. B. Rothbard, and B. A. Askonas, "Enhanced recognition of a modified peptide antigen by cytotoxic T cells specific for influenza nucleoprotein," Cell, vol. 52, no. 2, pp. 253-258, 1988.

[22] L. Ramakrishna, K. K. Anand, K. M. Mohankumar, and U. Ranga, "Codon optimization of the Tat antigen of human immunodeficiency virus type 1 generates strong immune responses in mice following genetic immunization," Journal of Virology, vol. 78, no. 17, pp. 9174-9189, 2004.

[23] A. Castaldello, E. Brocca-Cofano, R. Voltan et al., "DNA prime and protein boost immunization with innovative polymeric cationic core-shell nanoparticles elicits broad immune responses and strongly enhance cellular responses of HIV-1 tat DNA vaccination," Vaccine, vol. 24, no. 29-30, pp. 5655-5669, 2006.

[24] B. Garulli, G. di Mario, M. G. Stillitano, Y. Kawaoka, and M. R. Castrucci, "Exploring mucosal immunization with a recombinant influenza virus carrying an HIV-polyepitope in mice with pre-existing immunity to influenza," Vaccine, vol. 32, no. 21, pp. 2501-2506, 2014.

[25] G. Goldstein, "HIV-1 Tat protein as a potential AIDS vaccine," Nature Medicine, vol. 2, no. 9, pp. 960-964, 1996.

[26] B. Ensoli, V. Fiorelli, F. Ensoli et al., "Candidate HIV-1 Tat vaccine development: from basic science to clinical trials," AIDS, vol. 20, no. 18, pp. 2245-2261, 2006.

[27] A. Cafaro, A. Caputo, C. Fracasso et al., "Control of SHIV89.6P-infection of cynomolgus monkeys by HIV-1 Tat protein vaccine," Nature Medicine, vol. 5, no. 6, pp. 643-650, 1999.

[28] A. Cafaro, F. Titti, C. Fracasso et al., "Vaccination with DNA containing tat coding sequences and unmethylated CpG motifs protects cynomolgus monkeys upon infection with simian/human immunodeficiency virus (SHIV89.6P)," Vaccine, vol. 19, no. 2-22, pp. 2862-2877, 2001.

[29] M. T. Maggiorella, S. Baroncelli, Z. Michelini et al., "Longterm protection against SHIV89.6P replication in HIV-1 Tat 
vaccinated cynomolgus monkeys," Vaccine, vol. 22, no. 25-26, pp. 3258-3269, 2004.

[30] A. Borsetti, S. Baroncelli, M. T. Maggiorella et al., "Containment of infection in Tat vaccinated monkeys after rechallenge with a higher dose of SHIV89.6Pcy243," Viral Immunology, vol. 22, no. 2, pp. 117-124, 2009.

[31] F. Ferrantelli, M. T. Maggiorella, I. Schiavoni et al., "A combination HIV vaccine based on Tat and Env proteins was immunogenic and protected macaques from mucosal SHIV challenge in a pilot study," Vaccine, vol. 29, no. 16, pp. 2918-2932, 2011.

[32] Z.-N. Li, S. N. Mueller, L. Ye et al., "Chimeric influenza virus hemagglutinin proteins containing large domains of the Bacillus anthracis protective antigen: protein characterization, incorporation into infectious influenza viruses, and antigenicity," Journal of Virology, vol. 79, no. 15, pp. 10003-10012, 2005.

[33] T. Horimoto and Y. Kawaoka, "Reverse genetics provides direct evidence for a correlation of hemagglutinin cleavability and virulence of an avian influenza A virus," Journal of Virology, vol. 68, no. 5, pp. 3120-3128, 1994.

[34] S. Debaisieux, F. Rayne, H. Yezid, and B. Beaumelle, “The ins and outs of HIV-1 Tat," Traffic, vol. 13, no. 3, pp. 355-363, 2012.

[35] E. Peduzzi, N. Westerfeld, R. Zurbriggen, G. Pluschke, and C. A. Daubenberger, "Contribution of influenza immunity and virosomal-formulated synthetic peptide to cellular immune responses in a phase I subunit malaria vaccine trial," Clinical Immunology, vol. 127, no. 2, pp. 188-197, 2008.

[36] R. Schumacher, M. Adamina, R. Zurbriggen et al., "Influenza virosomes enhance class I restricted CTL induction through $\mathrm{CD}^{+} \mathrm{T}$ cell activation," Vaccine, vol. 22, no. 5-6, pp. 714-723, 2004.

[37] R. Zurbriggen and R. Glück, "Immunogenicity of IRIV- versus alum-adjuvanted diphtheria and tetanus toxoid vaccines in influenza primed mice," Vaccine, vol. 17, no. 11-12, pp. 1301-1305, 1999.

[38] C. Moser, M. Amacker, A. R. Kammer, S. Rasi, N. Westerfeld, and R. Zurbriggen, "Influenza virosomes as a combined vaccine carrier and adjuvant system for prophylactic and therapeutic immunizations," Expert Review of Vaccines, vol. 6, no. 5, pp. 711721, 2007. 



Submit your manuscripts at

http://www.hindawi.com

Journal of
Signal Transduction
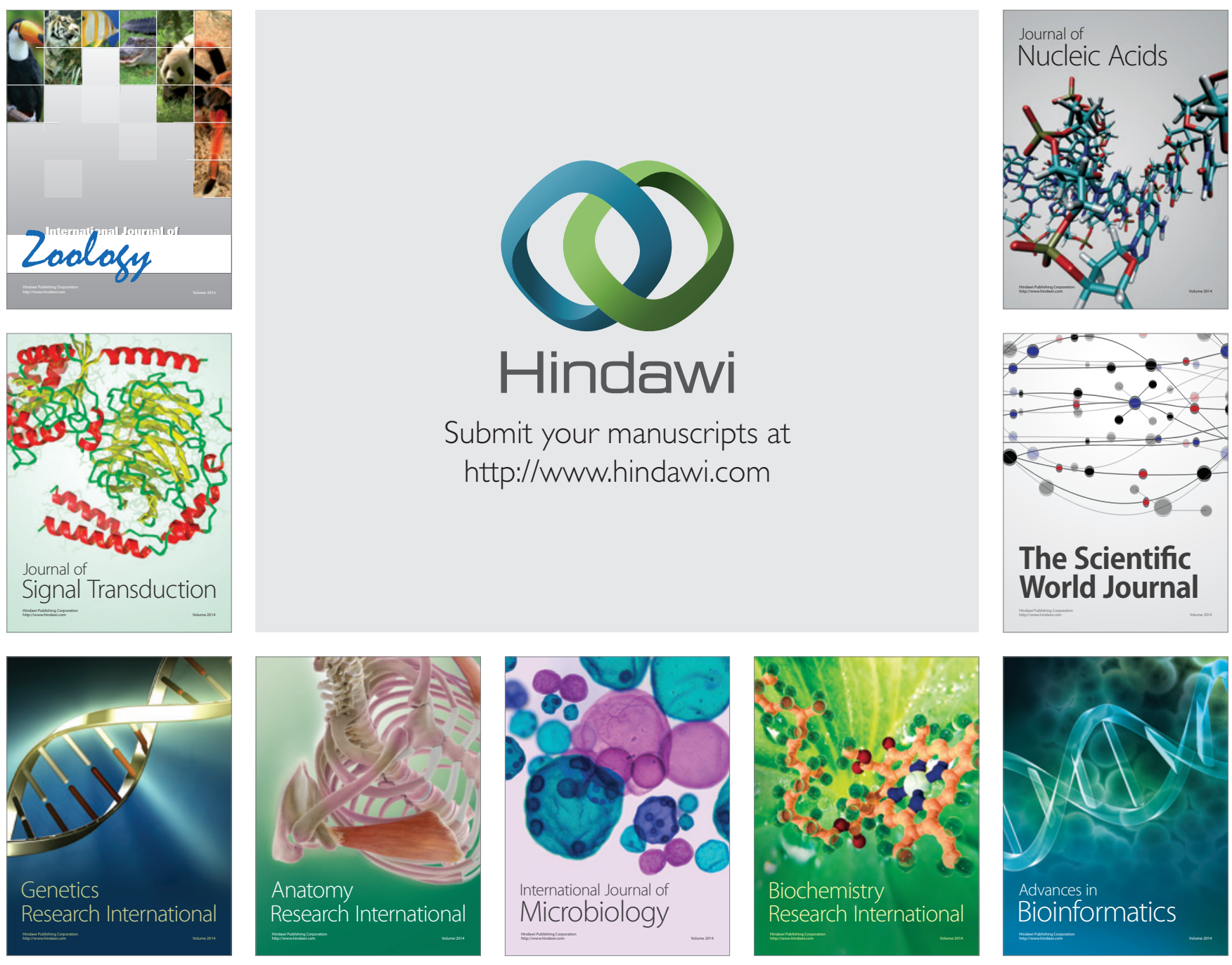

The Scientific World Journal
